\title{
Kernos
}

Revue internationale et pluridisciplinaire de religion grecque antique

13 | 2000

Varia

\section{Maddalena L. ZUNINO, Hiera Messeniaka. La storia religiosa della Messenia dall'età micenea all'età ellenistica}

Madeleine Jost

\section{(2) OpenEdition}

\section{Journals}

Édition électronique

URL : http://journals.openedition.org/kernos/1312

DOI : 10.4000/kernos. 1312

ISSN : 2034-7871

Éditeur

Centre international d'étude de la religion grecque antique

Édition imprimée

Date de publication : 1 janvier 2000

ISSN : 0776-3824

Référence électronique

Madeleine Jost, « Maddalena L. zunino, Hiera Messeniaka. La storia religiosa della Messenia dall'età micenea all'età ellenistica », Kernos [En ligne], 13 | 2000, mis en ligne le 16 juin 2011, consulté le 24 septembre 2020. URL : http://journals.openedition.org/kernos/1312 ; DOI : https://doi.org/10.4000/ kernos. 1312 
G. Ekroth examine les sites consacrés aux héros, depuis les plus anciens (viI ${ }^{\mathrm{e}}$ siè cle) jusqu'à ceux de l'époque hellénistique. Elle remet en question la pertinence du sens donné au vocabulaire relatif aux sites consacrés aux héros (notions d'eschara, d'holocauste, ...) et montre que ces définitions élaborées par les auteurs d'époque hellénistique ne correspondent pas aux réalités archéologiques des sites anciens. J. Binder reprend d'un point de vue critique la thèse adoptée par Mylonas selon laquelle le culte de Déméter à Éleusis remonterait à 1500 avant J.C.. Réexaminant les témoignages archéologiques (stratigraphiques et céramologiques), elle démontre que le premier centre cultuel ne pourrait pas être daté antérieurement au $\mathrm{vil}^{\mathrm{e}}$ siècle, date qui correspond aussi à la datation du noyau originel de l'Hymne bomérique à Déméter. Deux contributions enfin se concentrent sur des sites très différents : à Amorgos, une équipe dirigée par L. Marangou a récemment mis au jour un sanctuaire d'époque archaïque. L'A. propose d'y voir un lieu de culte consacré au héros-fondateur mythique de la cité; les témoignages plus tardifs, d'époque classique et hellénistique, semblent se rattacher au monde dionysiaque, une inscription mentionnant même le nom de Dionysos. L'autre site est celui d'Éphèse: A. Bammer tente d'y reconstituer le déroulement du rituel entre le vin ${ }^{\mathrm{e}}$ et le vi $\mathrm{v}^{\mathrm{e}}$ siècle. Cette démarche est aussi celle que propose la contribution de $\mathrm{N}$. Bookidis à propos du sanctuaire de Déméter et de Koré à Corinthe où furent mises au jour des tablettes de malédiction, et pour lesquelles l'A. propose un rapprochement avec la description d'un rituel attesté en Lydie.

Ces contributions montrent quel profit l'historien de la religion aurait à confronter systématiquement les données littéraires et iconographiques aux réalités du terrain archéologique. À l'exception de deux articles (ceux d'A. Bammer et d'E. Gebhard), peu d'auteurs présentent des stratigraphies des sites, précaution qui eût été utile quand il s'agit d'apprécier les particularités d'un locus bien déterminé (ex. cella de temple) où la localisation exacte des objets est déterminante, voire sur des sites dont les auteurs s'attachent à mettre en évidence les phénomènes de persistance. Trois index : général, des noms d'auteurs antiques, épigraphique.

Isabelle Tassignon (Université de Liège)

Maddalena L. Zunino, Hiera Messeniaka. La storia religiosa della Messenia dall'età micenea all'età ellenistica, Udine, Forum S.r.1., 1997. 1 vol. $17 \times 24$ cm, 379 p. ill. (Fonti e Testi. Raccolta di Storia e Filologia). ISBN : 88-8675660-5.

L'ouvrage de M. Zunino est une somme sur les cultes messéniens. On y trouve en effet, pour chaque chapitre consacré à un ensemble de divinités ou à un héros, un catalogue des testimonia littéraires, épigraphiques et numismatiques, depuis les tablettes de Pylos en linéaire B jusqu'à Pausanias (ce dernier est utilisé pour les informations sur le passé local qu'il donne, et non comme un reflet de son époque, car l'étude n'inclut pas l'époque romaine). Les sources sont classées suivant leur nature, le site et l'époque concernés; les textes sont traduits. Suit, pour chaque personnalité du culte, un essai de synthèse. Les données de l'archéologie ne sont prises en compte qu'au cours de ce dernier développement : elles sont souvent tenues pour secondaires, dans la mesure où leur témoignage n'est pas antérieur au $\mathrm{VIII}^{\mathrm{e}} / \mathrm{VII}^{\mathrm{e}} \mathrm{s}$.

Une idée sous-tend l'ensemble de l'étude. L'histoire religieuse messénienne est marquée par une longue période d'âges obscurs : les Dark Ages, puis la période de la domination laconienne entre le $\mathrm{viI}^{\mathrm{e}}$ et le $\mathrm{IV}^{\mathrm{e}}$ siècle. Pendant cette période, les hilotes messéniens ont conservé quelque chose de leur identité reli- 
gieuse, si bien que le panthéon en honneur après la restauration de la cité de Messène en 370 n'est pas une création du $\mathrm{IV}^{\mathrm{e}}$ siècle; il résulte de la " systématisation d'un patrimoine dispersé, mais ancien et messénien " (p. 29).

L'auteur commence par étudier les dieux. Le sujet du premier chapitre découle de la conception d'ensemble qu'a l'auteur du panthéon messénien. Artémis et Dionysos sont considérés comme les témoins d'une koiné messénolaconienne antérieure à la première guerre messénienne et qui pourrait remonter à l'époque mycénienne : le nom d'Artémis est attesté en mycénien et Artémis Limnatis, par exemple, devait être honorée à Limnai, à la frontière des deux régions, dans un culte commun fort ancien dont la légende du viol des jeunes filles spartiates (jeunes gens déguisés) par les Messéniens, ainsi que le meurtre du roi Téléklos, traduirait à la fois l'antiquité et le caractère initiatique. Artémis Orthia présente un autre aspect de I'Artémis de Limnai; l'A. étudie aussi le lieu de culte d'Artémis Oupésia à Messène et le sacerdoce de la déesse qui descendrait de Kresphontès, ce qui assure l'ancienneté du culte; elle reconstitue des aspects possibles de ce culte (initiation réservée aux parthénoi du génos des descendants de Kresphontès, avec xoanophorie, lampadédromie, sacrifice et banquet). Un développement sur Artémis Laphria fait une place à la communauté messénienne de Naupacte dans la constitution du panthéon du $\mathrm{IV}^{\mathrm{e}}$ siècle. Dionysos proviendrait lui aussi d'une koiné messéno-laconienne. Pour prouver l'ancienneté de son culte, qui a perdu de l'importance à l'époque historique, l'A. invoque l'existence du nom du dieu dans les tablettes mycéniennes (une attestation à Pylos) et l'absence de toute légende de « résistance » au culte de Dionysos en Messénie, ce qui montrerait l'ancienneté de son implantation.

Les chapitres suivants ( 2 à 4 ) sont organisés selon les fonctions des divinités. Sont étudiés le maître des sommets et son épouse (Zeus, Diwia et Héra), les divinités «vénérables » (Déméter, Koré et Poseidon), les divinités qui veillent sur la défense de la polts et la protection de l'individu (Athéna, Apollon et Asklépios). Au passage, l'A. souligne, en plus de ces aspects spécialisés, l'étendue des compétences de chaque divinité : ainsi des inscriptions votives d'euploia provenant de l'île de Proté attestent un lien privilégié de Zeus, de Déméter et d'Athéna avec la navigation. En ce qui concerne l'épiclèse de Zeus Sôter que l'auteur lie en premier lieu au salut individuel, son attestation à Mégalopolis et à Mantinée comme à Messène invite plutôt à privilégier l'idée d'une signification politique et patriotique. L'étude d'Apollon Korythos, dieu casqué et guerrier, donne lieu à d'intéressants développements sur le dieu «cornu» dans le monde grec depuis l'époque mycénienne. Concernant Asklépios, l'existence d'un culte d'Asklépios et Hygie Sôtéres et Poliouchoi dans l'apoikia de Zankle/Messana apporte un argument convaincant pour y voir, autant qu'un dieu guérisseur, une sorte de divinité poliade de Messène. Dans tous les cas évoqués dans ces chapitres, il s'agirait, selon l'A., de divinités qui ont un passé mycénien. Pour Héra, connue à Pylos mais qui n'est pas attestée en Messénie à l'époque historique, sa présence à Zankle/Messana laisserait supposer l'existence d'un culte aussi en Messénie, bien que Pausanias ne le mentionne pas. L'A. souligne la fréquence des cérémonies à mystères en Messénie : en l'honneur de Déméter, mais aussi d'Apollon, honoré avec son fils Dryops (p. 178).

Après avoir étudié quelques cas problématiques (chap. 5, Les Dioscures, les Kourètes, Eileithyia et les Kaloi Daimones), M.Z. consacre un chapitre aux dieux Pan et Hermès. Le dieu arcadien Pan n'a laissé que des traces en Messénie, mais il est largement attesté sur le monnayage de Messana entre 461 et 396 et peut provenir là de Messénie; quant à Hermès, son existence à Pharai d'Achaïe laisserait imaginer un culte similaire à Pharai de Messénie où Pan est l'objet de deux dédi- 
caces. Les autres cultes de Messénie, sur lesquels les données sont maigres, font l'objet du chapitre 7 (divinités mycéniennes, Aphrodite et Héphaistos, Thétis, Leukothéa, Tyché, les fleuves Achéloos et Pamisos, "les Dieux »), suivi de quelques pages sur les cultes orientaux. La seconde partie du livre, beaucoup plus brève (p. 251-286), traite des héros : héros nationaux (Messéné, Leukippos, Idès, Aristomène), Héraklès, héros guérisseurs et daimones (Tityros).

Au total, l'ouvrage donne une documentation très utile sur les panthéons mycénien et classico-hellénistique. Pour la période qui va de la chute de Pylos à la fondation de Messène, il propose des pistes (caractère ancien des légendes, attestations dans l'apoikia de Messana ou à Naupacte) qui peuvent laisser supposer l'existence de tel ou tel culte. On a là, malgré les incertitudes qui pèsent parfois sur les analyses de détail, un ouvrage particulièrement utile.

\section{Madeleine Jost \\ Madeleine Jost}

Marcel Detienne, Apollon le couteau à la main. Une approche expérimentale du polytbéisme grec, Paris, Gallimard, 1998. 1 vol. $14 \times 22,5 \mathrm{~cm}, 350$ p. (Bibliotbèque des Sciences bumaines). ISBN : 2-07-073371-8.

À défaut de pouvoir encore effrayer, ce titre insolite, qu'illustre une magnifique représentation sur céramique du peintre de Penthésilée, est sans nul doute destiné à surprendre. Mais là n'est pas sa seule raison d'être. L'A. s'en explique dans son «Ouverture " (Apollon, pourquoi pas ?). Il s'agit de redresser une image édifiante et bien ennuyeuse du dieu que, depuis Winckelmann, relayé notamment par W. Otto dans Les Dieux de la Grèce, l'érudition moderne n'a cessé de véhiculer : « décrire Apollon, écrivait le premier, exige le style le plus haut : une élévation au-dessus de tout ce qui est humain ». On savait $M$. Detienne très enclin, au départ de ses ouvrages, à se donner des cibles pour mieux marquer sa différence. Sa critique est ici très mesurée dans le ton, eu égard sans doute à la qualité re connue par ailleurs à cet ouvrage d'Otto dont il a préfacé la traduction française. Il n'empêche que, sous le couvert plus discret des notes, certains philologues sont davantage étrillés; ainsi J. Defradas est-il soupçonné d'avoir, dans son interpré tation de la mort de Néoptolème, transformé "Apollon en Sacré-Cour, au-delà du Dieu moral qui a la peau si dure dans les évocations de la Grèce immortelle, entre académiciens de tout sexe » (p. 315, n. 77)!

L'humour, n'est pas déplacé quand on disserte de dieux au rire inextinguible, et il est rare, reconnaissons-le, de le découvrir ici aussi grinçant. Du moins met-il bien le doigt sur ce qui insupporte particulièrement l'A. : le parti-pris de gommer ou d'épurer, contre l'évidence parfois, ce qui, dans les sources anciennes, viendrait à contredire l'image qu'on s'est faite d'un dieu parfait de la lumière et de la sagesse. Si M. Detienne a choisi pour sa part de mettre en relief telle face noire du Delphien, en le " prenant de biais, par surprise, de nuit ", ce n'est pas, explique-til, pour l'affronter à sa clarté, mais pour rendre compte plus fidèlement, en suivant ses gestes et ses pratiques, de " la texture d'un système polythéiste dont il fait partie » au même titre que les autres Olympiens.

Le sous-titre annonce clairement ce programme méthodologique au sujet duquel l'auteur s'est d'ailleurs expliqué plus longuement dans un récent numéro de Kernos $(10,1997$, p. 57-72). Il s'agit de pratiquer « la micro-analyse d'une coupe prélevée dans ce tissu à dieux multiples ». G. Dumézil est, dans cette perspective, crédité d'un double apport original dont on peut encore aujourd'hui s'inspirer : d'une part, il s'est montré attentif aux groupements récurrents ou circonstanciels faisant apparaître des complémentarités, des antithèses, des hiérarchies; d'autre part, il a souligné le fait qu'en régime polythéiste, un dieu ne peut 